\title{
Ética de la Función Pública y mejora de gestión pública del Gobierno Regional de
} Apurímac

\section{Ethics of the Public Function and improvement of public management of the Apurimac Regional Government}

\author{
Eleuterio Morales ${ }^{1}$
}

\section{RESUMEN}

Objetivo: El objetivo de la investigación es determinar la influencia del Código de Ética de la Función Pública en la mejora de la Gestión Pública de la sede central del Gobierno Regional de Apurímac 2018 Método: A través del método cuantitativo, de tipo básica, no experimental transversal de nivel descriptivo correlacional, se empleó una encuesta estadísticamente determinada por 132 servidores compuesto por personal nombrado y contratado, mediante un cuestionario estructurado validado por juicio de expertos y analizados mediante la fiabilidad de la escala Alpha de Cronbach. Para la comprobación de la hipótesis se utilizó el análisis de correlación Spearman mediante el software estadístico IBM SPSS, versión 22. Resultados: el 4.5\% de encuestados expresan que siempre aplican el Código de Ética de la Función Pública y el 43.2\% de encuestados manifiestan que casi nunca existe la mejora en la Gestión Pública y a través del coeficiente de correlación de Spearman a un nivel de confianza al 95\% $($ sig $=.00)$ de determina que existe una correlación positiva alta $(\mathrm{r}=0,899)$ entre el código de ética de la función pública y la mejora de la gestión, y correlación directa positiva alta $(r=0,899)$ entre la dimensión de los principios éticos del servidor público y mejora de la Gestión Pública; correlación positiva muy alta $(\mathrm{r}=0,927)$ entre la dimensión de los deberes éticos del servidor público y mejora de la Gestión Pública ; una correlación positiva alta ( $\mathrm{r}=0,898)$ entre la dimensión de las prohibiciones éticas del servidor público y la mejora de la Gestión Pública en la Sede Central del Gobierno Regional de Apurímac 2018. Conclusión: Existe una relación significativa entre código de ética de la función pública y la mejora la gestión pública, por lo tanto entonces es importante aplicar el código de ética para ofrecer servicios de calidad a los usuarios, y evitar la corrupción.

Palabras clave: código, ética, principios, deberes, prohibiciones, función pública.

1. Doctor en Ciencias Empresariales - Universidad Nacional de san Agustín, Magister en Administración - Universidad Nacional de San Antonio abad del Cusco, Diplomado - Centro de Capacitación Profesional / Emprendimiento y Desarrollo Empresarial - Metodología de la Investigación. Email: eloymorale@hotma0il.com 
The research entitled "The Code of Ethics of Public Function and Improvement of Public Management of the Headquarters of the Regional Government of Apurimac 2018", whose objective was: To determine how the Code of Ethics of the Public Function influences the improvement of Public Management of the central headquarters of the Regional Government of Apurímac 2018 Methods: It is framed within quantitative research, of a basic, non-experimental cross-sectional type of correlational descriptive level. A statistically determined sample survey of 132 public servants appointed and hired was used, using a structured questionnaire validated by expert judgment and analyzed by the reliability of the Cronbach Alpha scale and for the verification of the hypothesis the Spearman correlation analysis was used. Using the IBM SPSS statistical software, version 22; Results: $4.5 \%$ of respondents express that they always apply the Code of Ethics of Public Service and $43.2 \%$ of respondents say that there is almost never an improvement in Public Management and Spearman's correlation coefficient of 95\% confidence level. \% obtaining result of level of significance ( $\operatorname{sig}=.00)$ that is less than $0.05(5 \%)$ that there is a high positive correlation $(\mathrm{r}=0.899)$ between the code of ethics of the public function and the improvement of Management, and high positive direct correlation $(r=0.899)$ between the dimension of the ethical principles of the public servant and improvement of Public Management; very high positive correlation $(r=0.927)$ between the dimension of the ethical duties of the public servant and improvement of Public Management; a high positive correlation $(\mathrm{r}=0,898)$ between the dimension of the ethical prohibitions of the public servant and the improvement of Public Management in the Headquarters of the Regional Government of Apurímac 2018 Discussion: There is a significant relationship between the code of ethics of the public service and the Public Management improvement then I infer that it is extremely important to practice the code of ethics of the public function to improve the public function to offer quality services to users. And avoid corruption.

Keywords: Code, Ethics, principles, duties, prohibitions, Public Function.

\section{INTRODUCCIÓN}

La presente investigación se refiere al tema del Código de Ética de la Función Pública y Mejora de la Gestión Pública de la Sede Central del Gobierno Regional de Apurímac 2018, y el Código de Ética de la Función Pública fue promulgado por el (Presidente Constitucional de la República , 2002) que definí como que los fines de la función pública son el Servicio a la Nación, de conformidad con lo dispuesto en la Constitución Política, y la obtención de mayores niveles de eficiencia del aparato estatal, de manera que se logre una mejor atención a la ciudadanía, priorizando y optimizando el uso de los recursos públicos y (Bustamante, 2006).

Define que la ética pública es el conjunto de objetivos o de fines que se consideran debe realizar el poder político a través de su Derecho, teniendo como meta el desarrollo integral de cada persona como la ética y al referirse al ámbito público se relaciona con la política y funcionarios públicos.

Por ende un buen gobierno no solo requiere funcionarios responsables sino también políticos responsables, puesto que son éstos últimos principalmente quienes gozan del máximo mar- 
gen de autonomía en las decisiones y de estas decisiones depende a su vez la actuación de los principios; y por otra parte sobre la Mejora de la Gestión Pública es a base de la eficiencia gubernamental y prevención de la corrupción (Gómez González, 2018) y (SFP.Secretaria de la Función Pública, 2013) define como la aplicación de todos los procesos e instrumentos propios de la administración pública para lograr los objetivos de desarrollo y de bienestar de la población; de ahí que la mejora de la Gestión Pública implica un mejor aprovechamiento de dichos procesos para maximizar la calidad de los trámites y servicios que presta la Administración Pública Federal, a fin de satisfacer las expectativas de los ciudadanos y por ende los procesos de mejoramiento de la Gestión Pública promueven homologación de métodos, adopción de buenas prácticas, eliminación de normas que entorpecen el quehacer gubernamental y el empleo de herramientas que fomentan el incremento de la efectividad y la disminución de costos de operación de las instituciones y a los efectos del presente Código, y también "se entiende por función pública toda actividad temporal o permanente, remunerada $u$ honoraria, realizada por una persona en nombre o al servicio de las entidades de la Administración Pública, en cualquiera de sus niveles jerárquicos" (Presidente Constitucional de la República, 2002).

Como antecedentes de trabajos previos se tiene de (Gallegos Llacta, 2016) titulado "La ética pública en el desempeño profesional de los funcionarios del Ministerio Público Sede Chiclayo 2016; (Garcìa Ortiz, 2012) "la ética en el desempeño de la administración pública, marco legal y su forma de aplicación en la función del estado y no existiendo estudios en el ámbito local.

La característica principal sobre el Código de Ética de la Función Pública es una Ley y cuyo Ámbito de aplicación son Los Principios, Deberes y Prohibiciones éticos que rigen para los servidores públicos de las entidades de la Administración Pública y la Gestión Pública es mejorar la calidad de servicio a los usuarios o bienestar social.
Para analizar esta problemática es necesario de mencionar sus causas. Una de ellas es que la población no percibe la mejora el desarrollo social, económico y la calidad de servicios en las diferentes las entidades de la Administración Pública como la sociedad sigue enfrentando la baja valoración de nuestras autoridades Nacionales, Regionales y locales, como la corrupción que quebranta las bases institucionales y la legitimidad de los tres niveles de gobierno, y a lo largo del tiempo se ha implementado un sistema normativo que viene regulando la conducta ética de los servidores públicos como es la Ley del Código de Ética de la Función Pública Ley No 27815 promulgado por el (Presidente Constitucional de la República, 2002) con la finalidad de que la función pública esté al Servicio a la Nación y con obtención de mayores niveles de eficiencia del aparato estatal para que se logre una mejor atención a la ciudadanía, priorizando y optimizando el uso de los recursos públicos conforme a lo dispuesto por la Ley Marco de Modernización de la Gestión del Estado, sin embargo la población no perciben la mejora de la gestión pública más a lo contrario perciben deficiente gestión pública como beneficios de carácter particular, influencias, beneficios particulares que involucran al funcionario público.

Por otra parte el Instituto de Estadística e Informática (INEI), (Costa Aponte, Sánchez Aguilar, Hidalgo Calle, \& Benavides Rullier, 2018) indica que la gestión en el Perú del gobierno central su capacidad y la eficacia de la dirección del gobierno y su acción en la administración pública, tiene muy baja valoración de la población en todas las instancias de gobierno lo cual se evidencia en los datos obtenidos entre Setiembre 2017 - Febrero 2018 , donde solo el $24,6 \%$ de encuestados opinan que la gestión del Gobierno Central es buena o muy buena. Y a nivel de Gobierno Regional es de $21,6 \%$ que califica de buena o muy buena la gestión del gobierno regional y un $28,9 \%$ en el mismo sentido por el gobierno local.

El Gobierno Regional de Apurímac es un Organismo Público descentralizado, con persone- 
ría jurídica de derecho público, con autonomía política, económica y administrativa en asuntos de su competencia, constituyendo, para su administración económica y financiera; cuya finalidad según la Ley orgánica de gobiernos regionales Ley No 27867 promulgado por el (Presidente Constitucional de la República, 2002) que tiene por finalidad esencial fomentar el desarrollo Regional integral sostenible, promoviendo la inversión pública y privada y el empleo y garantizar el ejercicio pleno de los derechos y la igualdad de oportunidades de sus habitantes, de acuerdo con los planes y programas nacionales, regionales y locales de desarrollo, sin embargo en el Gobierno Regional de Apurímac existe una baja valoración acerca de la Gestión Pública como lo evidencia en el INE según (Costa Aponte, Sánchez Aguilar, Hidalgo Calle, \& Benavides Rullier, 2018), en los datos obtenidos entre Setiembre 2017 - Febrero 2018 , donde el $65.6 \%$ de encuestados opinan que la gestión pública es mala o muy mala Además existe malestar de la población Apurimeña, por no Priorización y optimización en el uso de los recursos públicos, la Obtención de mayores niveles de Eficiencia y eficacia de prestación de servicios y no existe una mejor atención de calidad a la ciudadanía y en consecuencia el interés de conocer objetivo de esta investigación es de determinar de qué manera el código de ética de la función pública influye en la mejora de la Gestión Pública de la sede central del Gobierno Regional de Apurímac 2018, así como los objetivos específicos como son:

a) Determinar de qué manera los principios éticos del servidor público influye en la mejora de la Gestión Pública sede central del Gobierno Regional de Apurímac 2018;

b) Determinar de qué manera los deberes éticos del servidor público influye en la mejora de la Gestión Pública de la sede central del Gobierno Regional de Apurímac 2018;

c).-Determinar de qué manera las prohibiciones éticas del servidor público influye en la mejora de la Gestión Pública sede central del Gobierno
Regional de Apurímac 2018 , de la misma forma el interés académico de conocer cómo el código de ética de la función pública influye en la mejora de la Gestión Pública.

Y demostrar la hipótesis general y específica relacionada con el planteamiento de los objetivos de la investigación llevado en la sede central del Gobierno Regional de Apurímac 2018.

\section{MĖTODOS Y MATERIALES}

El tipo de estudio de la presente investigación es básica, descriptiva, de diseño de investigación no experimental de corte trasversal y correlacional; para levantar la información se aplicó la técnica de encuesta a través de un cuestionario previamente elaborada y validada, a una muestra poblacional aleatoria de 132 Servidores administrativos nombrados del gobierno Región de Apurímac calculada estadísticamente, de acuerdo al número de Servidores administrativos Nombrados, en base a un universo de 201 Servidores administrativos Nombrados

Para medir el conocimiento sobre ética de Función Pública y mejora de Gestión pública se elaboró un cuestionario de 19 preguntas para cada variable haciendo en total 38 preguntas, para la evaluación se utilizó la escala de Likert ("Nunca”, "Casi nunca", "Algunas veces", "Casi siempre”, "Siempre").

Con un índice de confiabilidad según Alfa de Cronbach para la variable de ética de Función Pública con valor de 0.989 (99\% de fiabilidad) y para la variable de mejora de Gestión pública 0,986(97\% de fiabilidad) respectivo que califica coeficiente alfa Cronbach de consistencia interna como excelente.

La investigación tuvo algunas limitaciones que al momento de la aplicación de la encuesta los servidores del gobierno regional contaban con sobre carga laboral, dificulto el proceso de levantamiento de la información. 
Tabla No 1 . Resultado estadístico mediante la tabla cruzada de código de ética y la mejora la Gestión Pública

\begin{tabular}{|c|c|c|c|c|c|c|c|c|}
\hline \multirow{14}{*}{$\begin{array}{l}\text { Código de } \\
\text { ética de la } \\
\text { Función } \\
\text { Pública }\end{array}$} & \multirow{4}{*}{ Nunca } & \multicolumn{7}{|c|}{ Mejora en la Gestión Publica } \\
\hline & & & Nunca & Casi nunca & $\begin{array}{l}\text { Algunas } \\
\text { veces }\end{array}$ & $\begin{array}{c}\text { Casi } \\
\text { siempre }\end{array}$ & Siempre & Total \\
\hline & & Recuento & 9 & 9 & 0 & 0 & 0 & 18 \\
\hline & & $\%$ del total & $6,80 \%$ & $6,80 \%$ & $0,00 \%$ & $0,00 \%$ & $0,00 \%$ & $13,60 \%$ \\
\hline & \multirow{2}{*}{ Casi nunca } & Recuento & 0 & 38 & 0 & 0 & 0 & 38 \\
\hline & & $\%$ del total & $0,00 \%$ & $28,80 \%$ & $0,00 \%$ & $0,00 \%$ & $0,00 \%$ & $28,80 \%$ \\
\hline & \multirow{2}{*}{ Algunas veces } & Recuento & 0 & 10 & 38 & 6 & 0 & 54 \\
\hline & & $\%$ del total & $0,00 \%$ & $7,60 \%$ & $28,80 \%$ & $4,50 \%$ & $0,00 \%$ & $40,90 \%$ \\
\hline & \multirow{2}{*}{ Casi siempre } & Recuento & 0 & 0 & 0 & 16 & 0 & 16 \\
\hline & & $\%$ del total & $0,00 \%$ & $0,00 \%$ & $0,00 \%$ & $12,10 \%$ & $0,00 \%$ & $12,10 \%$ \\
\hline & \multirow{2}{*}{ Siempre } & Recuento & 0 & 0 & 0 & 0 & 6 & 6 \\
\hline & & $\%$ del total & $0,00 \%$ & $0,00 \%$ & $0,00 \%$ & $0,00 \%$ & $4,50 \%$ & $4,50 \%$ \\
\hline & \multirow{2}{*}{ Total } & Recuento & 9 & 57 & 38 & 22 & 6 & 132 \\
\hline & & $\%$ del total & $6,80 \%$ & $43,20 \%$ & $28,80 \%$ & $16,70 \%$ & $4,50 \%$ & $100,00 \%$ \\
\hline
\end{tabular}

El $40.9 \%$ del total de encuestados manifiestan que algunas veces aplican el Código de ética de la Función Pública y el $4.5 \%$ de encuestados expresan que siempre lo aplican el Código de ética de la Función Pública; sin embargo el $43.2 \%$ de total de encuestados lo manifiestan que casi nunca existe la mejora en la Gestión Pública y solo el $4.5 \%$ de total de encuestados manifiestan que siempre existe la mejora en la Gestión Pública ,sin embrago un 28.8\% de total de encuestados expresan que alguna veces existe la mejora la Gestión Pública de la sede central del Gobierno Regional de Apurímac.

Tabla No 2. Resultado de correlación de hipótesis general de código de ética de la función pública y la mejora de la Gestión Pública de la sede central del Gobierno Regional de Apurímac 2018.

\begin{tabular}{|c|c|c|c|c|}
\hline & & & $\begin{array}{l}\text { Código de ética } \\
\text { de la Función } \\
\text { Pública }\end{array}$ & $\begin{array}{c}\text { Mejora en la } \\
\text { Gestión } \\
\text { Publica }\end{array}$ \\
\hline \multirow{6}{*}{$\begin{array}{c}\text { Rho de } \\
\text { Spearman }\end{array}$} & \multirow{3}{*}{$\begin{array}{l}\text { Código de ética } \\
\text { de la función } \\
\text { pública }\end{array}$} & $\begin{array}{l}\text { Coeficiente de } \\
\text { correlación }\end{array}$ & 1 & $899^{* *}$ \\
\hline & & Sig. (bilateral) & . & 0 \\
\hline & & $\mathrm{N}$ & 132 & 132 \\
\hline & \multirow{3}{*}{$\begin{array}{l}\text { Mejora en la } \\
\text { gestión } \\
\text { publica }\end{array}$} & $\begin{array}{l}\text { Coeficiente de } \\
\text { correlación }\end{array}$ &, $899^{* *}$ & 1 \\
\hline & & Sig. (bilateral) & 0 & . \\
\hline & & $\mathrm{N}$ & 132 & 132 \\
\hline
\end{tabular}

Los resultados revelaron que la variable de Código de ética de la Función Pública existe correlación positiva alta con la variable de la mejora en la Gestión Pública, según la correlación de Spearman de ,899 $(\mathrm{p}=0)$. 
Tabla No 3. Resultado estadístico mediante la tabla cruzada de la dimensión de principios éticos del servidor público y la mejora de la Gestión Pública sede central del Gobierno Regional de Apurímac 2018

\begin{tabular}{|c|c|c|c|c|c|c|c|c|}
\hline \multirow{14}{*}{$\begin{array}{l}\text { Principios } \\
\text { éticos del } \\
\text { servidor } \\
\text { público }\end{array}$} & \multirow{4}{*}{ Nunca } & \multicolumn{7}{|c|}{ Mejora en la gestión Pública } \\
\hline & & & \multirow{2}{*}{$\begin{array}{c}\text { Nunca } \\
9\end{array}$} & \multirow{2}{*}{$\begin{array}{c}\text { Casi nunca } \\
9\end{array}$} & \multirow{2}{*}{$\begin{array}{c}\text { Algunas } \\
\text { veces } \\
0\end{array}$} & \multirow{2}{*}{$\begin{array}{c}\text { Casi } \\
\text { siempre } \\
0\end{array}$} & Siempre & Total \\
\hline & & Recuento & & & & & 0 & 18 \\
\hline & & $\%$ del total & $6,80 \%$ & $6,80 \%$ & $0,00 \%$ & $0,00 \%$ & $0,00 \%$ & $13,60 \%$ \\
\hline & \multirow{2}{*}{ Casi nunca } & Recuento & 0 & 37 & 0 & 0 & 0 & 37 \\
\hline & & $\%$ del total & $0,00 \%$ & $28,00 \%$ & $0,00 \%$ & $0,00 \%$ & $0,00 \%$ & $28,00 \%$ \\
\hline & \multirow{2}{*}{ Algunas veces } & Recuento & 0 & 11 & 38 & 10 & 0 & 59 \\
\hline & & $\%$ del total & $0,00 \%$ & $8,30 \%$ & $28,80 \%$ & $7,60 \%$ & $0,00 \%$ & $44,70 \%$ \\
\hline & \multirow{2}{*}{ Casi siempre } & Recuento & 0 & 0 & 0 & 12 & 0 & 12 \\
\hline & & $\%$ del total & $0,00 \%$ & $0,00 \%$ & $0,00 \%$ & $9,10 \%$ & $0,00 \%$ & $9,10 \%$ \\
\hline & \multirow{2}{*}{ Siempre } & Recuento & 0 & 0 & 0 & 0 & 6 & 6 \\
\hline & & $\%$ del total & $0,00 \%$ & $0,00 \%$ & $0,00 \%$ & $0,00 \%$ & $4,50 \%$ & $4,50 \%$ \\
\hline & \multirow{2}{*}{ Total } & Recuento & 9 & 57 & 38 & 22 & 6 & 132 \\
\hline & & $\%$ del total & $6,80 \%$ & $43,20 \%$ & $28,80 \%$ & $16,70 \%$ & $4,50 \%$ & $100,00 \%$ \\
\hline
\end{tabular}

Del total de 132 encuestados; el 44.7\% manifiestan que algunas veces practican los principios éticos del Servidor Público y el $4.5 \%$ del total de encuestados expresan que siempre aplican los principios éticos del servidor público por otro lado el $43.2 \%$ de encuestados manifiestan que casi nunca existe la mejora en la gestión Pública y solo el $4.5 \%$ de total de encuestados manifiestan que existe la mejora en la gestión Pública de la sede central del Gobierno Regional de Apurímac.

Tabla No 4. Resultado de correlación del primer objetivo específico y la hipótesis específico de determinar de qué manera los principios éticos del servidor público influye en la mejora de la Gestión Pública sede central del Gobierno Regional de Apurímac 2018.

\begin{tabular}{|c|c|c|c|c|}
\hline & & & $\begin{array}{c}\text { Principios éticos } \\
\text { del servidor } \\
\text { público }\end{array}$ & $\begin{array}{l}\text { Mejora en la } \\
\text { gestión publica }\end{array}$ \\
\hline \multirow{6}{*}{$\begin{array}{l}\text { Rho de } \\
\text { Spearman }\end{array}$} & \multirow{3}{*}{$\begin{array}{c}\text { Principios } \\
\text { éticos del } \\
\text { servidor público }\end{array}$} & Coeficiente de & 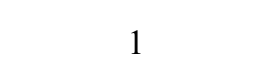 &, $878^{* *}$ \\
\hline & & Sig. (bilateral) & & 0 \\
\hline & & $\mathrm{N}$ & 132 & 132 \\
\hline & \multirow{3}{*}{$\begin{array}{l}\text { Mejora en la } \\
\text { gestión publica }\end{array}$} & $\begin{array}{l}\text { Coeficiente de } \\
\text { correlación }\end{array}$ &, $878^{* *}$ & 1 \\
\hline & & Sig. (bilateral) & 0 & . \\
\hline & & $\mathrm{N}$ & 132 & 132 \\
\hline
\end{tabular}

Como se puede ver en la tabla $\mathrm{N}^{\circ} 4$ el valor de "sig" es de 0.000 que es menor a 0.05 el nivel de significancia, entonces se rechaza la hipótesis nula (Ho); y acepta la hipótesis alterna (Hi) que los principios éticos del servidor público influye significativamente en la mejora de la Gestión Pública de la sede central del Gobierno Regional de Apurímac 2018. Con un nivel de confianza del 95\% que existe relación significativa entre la dimensión de los principios éticos del servidor público y la mejora en la Gestión Pública. Obteniendo el valor de correlación de spearman de 0,878 lo cual indica que existe una Correlación positiva alta. 
Tabla No 5. Resultados estadísticos de tabla cruzada entre la dimensión de deberes éticos del servidor público y la mejora de la Gestión Pública

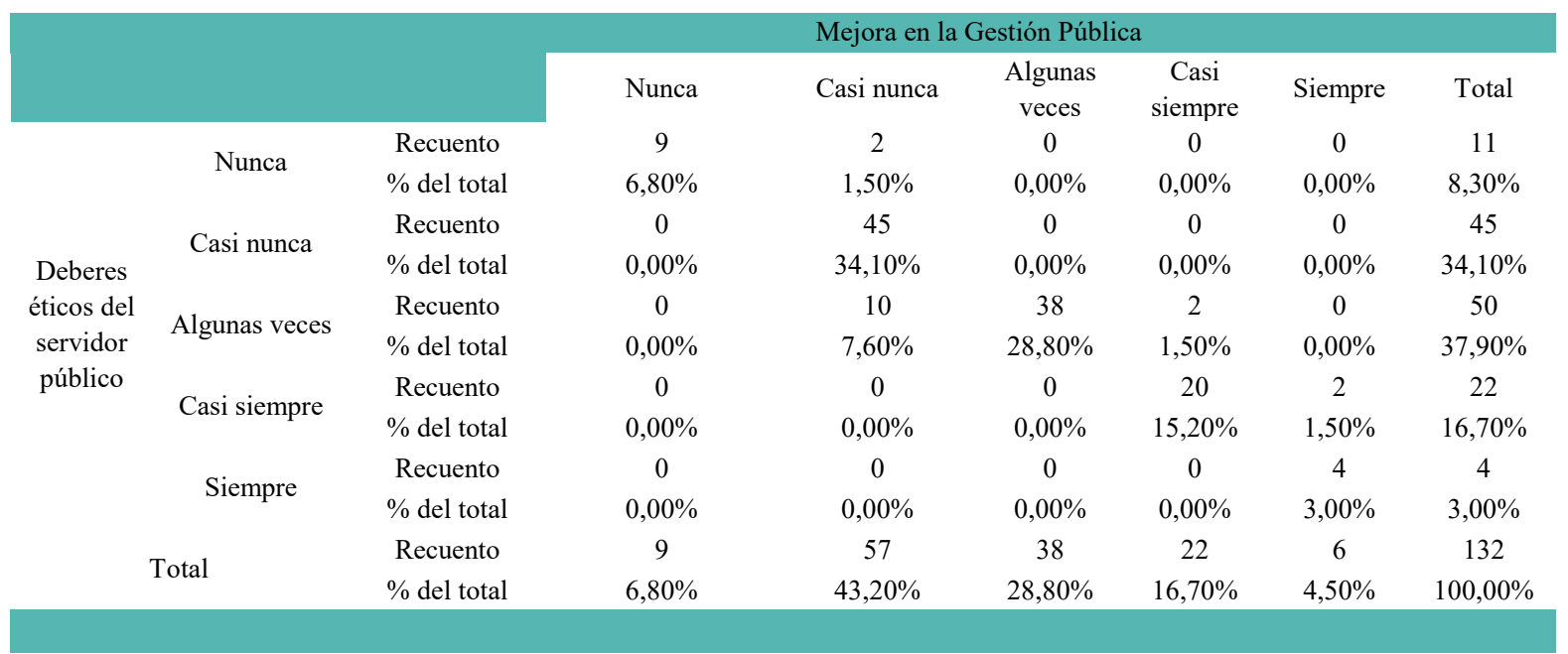

Del total de 132 encuestados; el 37.9\% de encuestados opinan que algunas veces aplican los deberes éticos del servidor público y solo el 3.0\% de total de encuestados expresan que siempre lo aplican los Deberes éticos del servidor público, por otro lado el $43.2 \%$ de total de encuestados expresan que casi nunca existe la mejora en la gestión pública y solo el $4.5 \%$ de total de encuetados opinan que siempre existe la mejora en la gestión Pública de la sede central del Gobierno Regional de Apurímac.

Tabla No 6 . Resultado de correlación del segundo objetivo específico de determinar de qué manera los deberes éticos del servidor público influye en la mejora de la Gestión Pública de la sede central del Gobierno Regional de Apurímac 2018.

\begin{tabular}{|c|c|c|c|c|}
\hline & & & $\begin{array}{l}\text { Deberes éticos del } \\
\text { servidor público }\end{array}$ & $\begin{array}{c}\text { Mejora en la } \\
\text { Gestión publica }\end{array}$ \\
\hline \multirow{6}{*}{$\begin{array}{l}\text { Rho de } \\
\text { Spearman }\end{array}$} & \multirow{3}{*}{$\begin{array}{l}\text { Deberes éticos } \\
\text { del servidor } \\
\text { público }\end{array}$} & $\begin{array}{l}\text { Coeficiente de } \\
\text { correlación }\end{array}$ & 1 &, $927^{* *}$ \\
\hline & & Sig. (bilateral) & . & 0 \\
\hline & & $\mathrm{N}$ & 132 & 132 \\
\hline & \multirow{3}{*}{$\begin{array}{c}\text { Mejora en la } \\
\text { gestión publica }\end{array}$} & $\begin{array}{l}\text { Coeficiente de } \\
\text { correlación }\end{array}$ &, $927^{* *}$ & 1 \\
\hline & & Sig. (bilateral) & 0 & · \\
\hline & & $\mathrm{N}$ & 132 & 132 \\
\hline
\end{tabular}

Los resultados revelaron que la variable de deberes éticos del servidor público influye significativamente en la mejora de la Gestión Pública obteniendo, el valor de "sig" de 0.000 que es menor a 0.05 el nivel de significancia, con un nivel de confianza del 95\% de una correlación de spearman de 0,927 lo que indica una correlación positiva muy alta. 
Tabla No 7 . Resultado de estadístico de tabla cruzada del tercer objetivo específico de prohibiciones éticas del servidor público y la mejora de la Gestión Pública

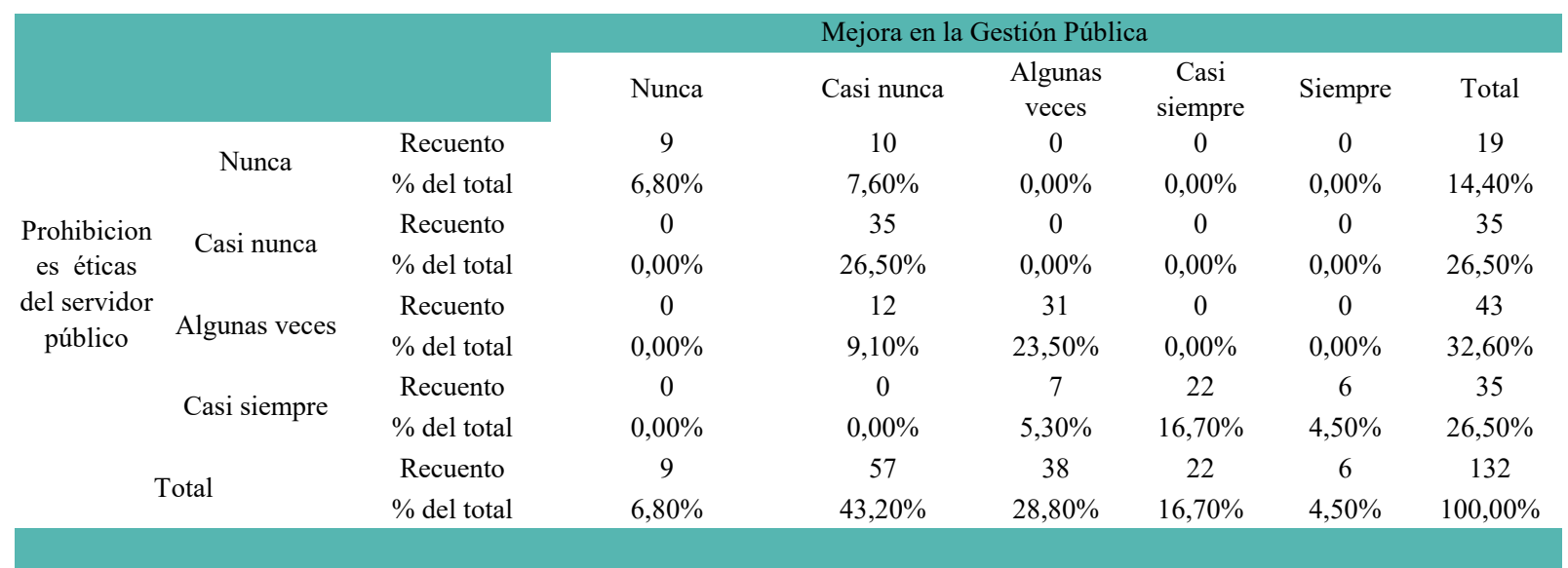

Del total de 132 encuestados; el 32.6\% expresan que algunas veces lo aplican las prohibiciones éticas del servidor público y solo el $26.5 \%$ de total de encuestados manifiestan que casi siempre lo aplican las prohibiciones éticas del servidor público mientras por otro lado el $43.2 \%$ de total de encuestados manifiestan que casi nunca existe la mejora en la Gestión Pública y solo el $4.5 \%$ expresan que siempre existe la mejora en la gestión Pública de la sede central del Gobierno Regional de Apurímac.

Tabla No 8. Resultado de correlación del tercer objetivo específico de determinar de qué manera las prohibiciones éticas del servidor público influye en la mejora de la Gestión Pública sede central del Gobierno Regional de Apurímac 2018

\begin{tabular}{|c|c|c|c|c|}
\hline & & & $\begin{array}{c}\text { Prohibiciones } \\
\text { éticas del servidor } \\
\text { público }\end{array}$ & $\begin{array}{c}\text { Mejora en la } \\
\text { Gestión Publica }\end{array}$ \\
\hline \multirow{6}{*}{$\begin{array}{c}\text { Rho de } \\
\text { Spearman }\end{array}$} & \multirow{3}{*}{$\begin{array}{c}\text { Prohibiciones } \\
\text { éticas del } \\
\text { servidor público }\end{array}$} & Coeficiente de & 1 &, $898^{* *}$ \\
\hline & & Sig. (bilateral) & & 0 \\
\hline & & $\mathrm{N}$ & 132 & 132 \\
\hline & \multirow{3}{*}{$\begin{array}{l}\text { Mejora en la } \\
\text { gestión publica }\end{array}$} & $\begin{array}{l}\text { Coeficiente de } \\
\text { correlación }\end{array}$ &, $898^{* *}$ & 1 \\
\hline & & Sig. (bilateral) & 0 & . \\
\hline & & $\mathrm{N}$ & 132 & 132 \\
\hline
\end{tabular}

Como se puede ver en la tabla 8 el valor de "sig" es de 0.000 que es menor a 0.05 de nivel de significancia, entonces se rechaza la hipótesis nula (Ho); por tanto se puede afirmar que las prohibiciones éticas del servidor público influye significativamente en la Gestión Pública de la sede central del Gobierno Regional de Apurímac 2018 con un nivel de confianza del 95\% y se demuestra que existe una relación significativa entre la dimensión de las prohibiciones éticas del servidor público y Gestión Pública Además la correlación de spearman es 0,898 lo que indica una Correlación positiva alta. 


\section{DISCUSIÓN}

Partiendo de la primicia que la ética se compone de un conjunto de juicios y de reglas que sirve para orientar nuestro comportamiento en la Vida, como una autoridad interna por la que regularizamos nuestros actos y juzgamos tanto nuestra conducta como de las demás (Pintos Santiago, 2017).

En la sede central del Gobierno Regional de Apurímac del año 2018 aplican mínimamente el Código de ética de la Función Pública y no existe mejoramiento de gestión pública a pesar que existe un sistema normativo formal, que con precisión y coherencia técnica regula la conducta ética de los servidores públicos que es Ley del Código de Ética de la Función Pública Ley No 27815 como indica los siguientes datos obtenidos en la Tabla 1 que solo el $4.5 \%$ de encuestados expresan que siempre lo aplican el Código de Ėtica de la Función Pública y el 40.9 \% del total de encuestados manifiestan que algunas veces aplican el Código de Ėtica de la Función Pública y por el otro lado sobre la mejora en la gestión pública el $43.2 \%$ de total de encuestados lo manifiestan que casi nunca existe la mejora en la gestión pública y solo el $4.5 \%$ de total de encuestados manifiestan que siempre existe la mejora en la Gestión Pública ,sin embrago un $28.8 \%$ de total de encuestados expresan que alguna veces existe la mejora la gestión pública.

Y al resultado del análisis inferencial logra establecer significancia estadística o la prueba de hipótesis tal como se demuestra en la tabla 2 cuyo valor de "sig" es de 0.000 que es menor a 0.05 de nivel de significancia, con un nivel de confianza del 95\% que existe una relación significativa entre la dimensión del código de ética de la función pública y la mejora la Gestión Pública con una correlación de spearman de, 0.899**, determinando que existe correlación positiva alta entonces podemos inferir que es sumamente importante practicar el código de ética de la función pública para mejorar la función pública, así como lo demostró también la investigación realizada por (Gallegos Llacta, 2016) titulado "La ética pública en el desempeño profesional de los funcionarios del Ministerio Público Sede Chiclayo 2016 ".

Y en cuanto al Primer objetivo específico: sobre la relación que existen entre la dimensión de los principios éticos del servidor público y la mejora de la Gestión Pública de la sede central del Gobierno Regional de Apurímac 2018. Podemos manifestar que mínimamente aplican o practican los principios de la Ley del Código de Ética de la Función Pública Ley No 27815 que enmarca en el Artículo 6 como el Respeto, Probidad, eficiencia, Idoneidad, Veracidad, Lealtad y Obediencia, Justicia y Equidad y Lealtad al Estado de Derecho como se indica en la tabla 3 que el $4.5 \%$ del total de encuestados expresan que siempre aplican los principios éticos del servidor público que es mínimo de funcionarios y de total de 132 encuestados el $44.7 \%$ manifiestan que algunas veces practican los principios éticos del Servidor Público, y en relación con la mejora en la gestión Pública el 43.2\% de encuestados manifiestan que casi nunca existe la mejora en la gestión Pública y solo el $4.5 \%$ de total de encuestados afirman que existe la mejora en la gestión Pública de la sede central del Gobierno Regional de Apurímac.

El resultado del análisis inferencial logra establecer significancia estadística o sometida a la prueba de hipótesis tal como se demuestra en la tabla 4 el valor de "sig" es de 0.000 que es menor a 0.05 el nivel de significancia, entonces se rechaza la hipótesis nula (Ho); por tanto se puede afirmar con un nivel de confianza del 95\% existe relación significativa entre la dimensión de los principios éticos del servidor público y mejora de la Gestión Pública.

Además la correlación de spearman es 0,878 lo cual indica que existe una Correlación positiva alta. Entonces podemos inferir que la aplicación de los principios de ética mejoraría significativamente la gestión pública. 
Y sobre el segundo objetivo específico: Sobre la relación que existen entre la dimensión de los deberes éticos del servidor público y la mejora de la Gestión Pública de la sede central del Gobierno Regional de Apurímac 2018. que los servidores cumplen mínimamente la Ley del Código de Ética de la Función Pública Ley No 27815 que enmarca en el Artículo 7 como la Neutralidad, Transparencia; Discreción; Ejercicio Adecuado del Cargo, Uso Adecuado de los Bienes del Estado, Responsabilidad, como los siguientes de Tabla 5 lo indican que del total de 132 encuestados; solo el 3.0\% de total de encuestados expresan que siempre lo aplican los deberes éticos del servidor público y el $37.9 \%$ de encuestados opinan que algunas veces aplican los deberes éticos del servidor público, por otro lado sobre la mejora en la gestión pública el $43.2 \%$ de total de encuestados expresan que casi nunca existe la mejora en la gestión pública y solo el $4.5 \%$ de total de encuetados opinan que siempre existe la mejora en la gestión pública que es lo mínimo; $\mathrm{Y}$ al resultado del análisis inferencial logra establecer significancia estadística o sometida a la prueba de hipótesis tal como se demuestra en la tabla 6 el valor de "sig" es de 0.000 que es menor a 0.05 el nivel de significancia, entonces se rechaza la hipótesis nula (Ho); por tanto se puede afirmar con un nivel de confianza del 95\% que existe relación significativa entre la dimensión de los deberes éticos del servidor público y la mejora la Gestión Pública; de correlación de spearman con un valor de 0,927 lo que indica una correlación positiva muy alta. Entonces podemos inferir que la aplicación de los deberes éticos del servidor público mejoraría significativamente la gestión pública.

Y por último del Tercer objetivo específico: sobre la relación que existe entre la dimensión de las prohibiciones éticas del servidor público y la mejora de la Gestión Pública de la sede central del Gobierno Regional de Apurímac 2018. Podemos decir que los servidores cumplen mínimamente la Ley del Código de Ética de la Función Pública Ley No 27815 que enmarca en el Artículo 8, como es de Mantener Intereses de Conflicto,
Obtener Ventajas Indebidas, Realizar Actividades de Proselitismo Político, Hacer Mal Uso de Información Privilegiada, Presionar, Amenazar y/o Acosar. como se indica en la Tabla 7, Que del total de 132 encuestados el 32.6\% expresan que algunas veces lo aplican las prohibiciones éticas del servidor público y solo el $26.5 \%$ de total de encuestados manifiestan que casi siempre lo aplican las prohibiciones éticas del servidor público mientras por otro lado sobre la mejora en la Gestión Pública el 43.2\% de total de encuestados manifiestan que casi nunca existe la mejora en la Gestión Pública y solo el $4.5 \%$ afirman que siempre existe la mejora en la gestión Pública de la sede central del Gobierno Regional de Apurímac; $\mathrm{Y}$ al resultado del análisis inferencial logra se establecer significancia estadística o sometida a la prueba de hipótesis tal como se demuestra en la tabla 8 con el valor de "sig" es de 0.000 que es menor a 0.05 con nivel de significancia, entonces se rechaza la hipótesis nula (Ho); por tanto se puede afirmar con un nivel de confianza del 95\% que existe relación significativa entre la dimensión de las prohibiciones éticas del servidor público y Gestión Pública con una correlación de spearman de 0,898 lo que indica una Correlación positiva alta. . Entonces podemos inferir que la aplicación de las prohibiciones éticas del servidor público mejoraría significativamente la gestión pública.

\section{CONCLUSIONES}

Se ha determinado con coeficiente de correlación de Spearman con un nivel de confianza al 95\% y de nivel de significancia de (sig $=.00)$ que es menor que del $0,05(5 \%)$ que existe una correlación positiva alta $(r=0,899)$ entre el código de ética de la función pública y la mejora de la Gestión Pública de la sede central del Gobierno Regional de Apurímac 2018.

Que existe una correlación directa positiva alta $(\mathrm{r}=0,899)$ de nivel de significancia de $($ sig $=.00)$ que es menor que del 0,05 (5\%) entre la dimensión de los principios éticos del servidor público y mejora de la Gestión Pública. 
Que existe una correlación positiva muy alta $(\mathrm{r}=0,927)$ de nivel de significancia de $($ sig $=.00)$ que es menor que del 0,05 (5\%) entre la dimensión de los deberes éticos del servidor público y mejora de la Gestión Pública.

Existe una correlación positiva alta $(\mathrm{r}=0,898)$ de nivel de significancia de $($ sig $=.00)$ que es menor que del 0,05 (5\%) entre la dimensión de las prohibiciones éticas del servidor público y la mejora de la Gestión Pública

Aunque no existe estudios sobre Código de ética de la Función Pública y el mejoramiento de gestión pública; existe un estudio titulado Prohibiciones éticas y tipos de personalidad en servidores públicos realizado en la Municipalidad Distrital de Pillco Marca de la provincia de Huánuco 2014, realizado por el autor (Salazar Sánchez, 2014) y cuyos resultados estadísticos apoyan a que existe una relación significativa entre prohibiciones éticas y tipos de personalidad.

Y de (Gallegos Llacta, 2016) concluye que la aplicación de la ética pública fortalece significativamente el desempeño profesional de los funcionarios del Ministerio Público sede Chicla, de la misma forma (Garcìa Ortiz , 2012)“.

La ética en el desempeño de la administración pública, marco legal y su forma de aplicación en la función del estado, su estudio de la ética pública responde a una de las tres cualidades que los griegos consideraban debía tener cualquier individuo que aspirara a ocupar un cargo público: (a) Lealtad hacía la Constitución establecida, (b) Capacidad para el cargo, y (c) Virtud y Justicia metodología descriptiva; donde concluye que el $90 \%$ de funcionarios son corruptos, el $73 \%$ piden presión para funcionarios deshonestos y el 94\% de encuestados piden que se implemente un órgano especializado sancionador de funcionarios corruptos.

Y por último (Farfan Pimentel, 2015) la Ètica en la Función Pública de la UGEl No 05 de san Juan de Lurigancho, Perú 2015; concluye: (a) La ética en la administración pública pone en juego todo un conjunto de valores para ofrecer servicios de calidad a la comunidad.

(b) La ética pública es un instrumento fundamental para evitar la corrupción al elegir a los representantes más idóneos para gobernar.

(c) En la administración pública se realizan actividades de servicio a los ciudadanos.

\section{REFERENCIAS}

Bustamante, R. (2006). Un modelo de lo de interrelación entre la moral, el poder y el derecho. Lima: ARA Editore. Recuperado el 18 de Agosto de 2018

Cifuentes Torres, F. A. (2009). El Desafío ético en la Función Pública: El Caso de carabineros de Chile. Santiago de Chile: Universidad de Chile.

Farfan Pimentel , J. F. (2015). La Ética en la Función Pública de la UGEL No 05 de San Juan de Lurigancho, Perú. Lima.

Gallegos Llacta, R. T. (2016). La ética pública en el desempeño profesional de los funcionarios del ministerio público sede Chiclayo 2016. Chiclayo: Universidad Cesar Vallejo.

Garcìa Ortiz , D. J. (2012). La Eticaen el Desempenode la Administracion Publica, Marco Legal Y su forma de Aplicacion en la Funcion del Estado. Chiapas: Instituto de Estudios Superiores de Chiapas.

Gómez González, A. (03 de Mayo de 2018). El economista. com.mx. Obtenido de eleconomista.com.mx: https://www. eleconomista.com.mx/opinion/Mejora-de-la-gestion-publica-base-de-la-eficiencia-gubernamental-y-prevencionde-la-corrupcion.-20180504-0009.html

Pintos Santiago, J. (2017). Calidad,transparencia y etica publica. Lima: INAP. Recuperado el 15 de Setiembre de 2018, de http://publicacionesoficiales.boe.es

Presidente Constitucional de la República . (12 de Agosto de 2002). mef.gob.pe. Recuperado el 15 de Agosto de 2018, de mef.gob.pe: https://www.mef.gob.pe/es/correoinstitucional? $\mathrm{id}=3024$

Presidente Constitucional de la República. (22 de Julio de 2002). Ley del Código de Ética de la Función Pública Ley No 27815. Ley del Código de Ética de la Función Pública Ley No 27815. Lima, Peru, Peru. Recuperado el 5 de Mayo de 2018, de apps.contraloria.gob.pe: https://storage.servir. gob.pe/images/2013/11/Ley27815.pdf 
